Cayce Onks, DO, MS, ATC; Matthew Silvis, MD; Jayson Loeffert, DO; James Tucker, MD; Robert A. Gallo, MD Department of Family Medicine (Drs. Onks, Silvis, Loeffert, and Tucker) and Department of Orthopaedics (Drs. Onks, Silvis, Loeffert, Tucker, and Gallo), Penn State Hershey Medical Center

Bconks@pennstatehealth. psu.edu

The authors reported no potential conflict of interest relevant to this article.

doi: 10.12788/jfp.0156

\title{
Conservative or surgical management for that shoulder dislocation?
}

\author{
A number of factors-including patient age and risk for \\ recurrence-influence treatment choices. Here's a closer \\ look at what to consider.
}

\section{PRACTICE RECOMMENDATIONS}

> Start with conservative management of shoulder dislocation in patients older than 30 years and those with uncomplicated injuries. B

> Discourage strict immobilization; its utility is debated and it may not change outcomes. B

> Recommend a progressive rehabilitative program after the initial acute shoulder injury. B

> Consider surgical management for patients younger than 30 years who have complicated injuries with bone or cartilage loss, rotator cuff tears, or recurrent instability or for the highly physically active individual. B

Strength of recommendation (SOR)

A Good-quality patient-oriented evidence

B Inconsistent or limited-quality patient-oriented evidence

C Consensus, usual practice, opinion, disease-oriented evidence, case series
7 he shoulder, or glenohumeral joint, is the most commonly dislocated large joint; dislocation occurs at a rate of 23.9 per 100,000 person/years. ${ }^{1,2}$ There are 2 types of dislocation: traumatic anterior dislocation, which accounts for roughly $90 \%$ of dislocations, and posterior dislocation $(10 \%){ }^{3}$ Anterior dislocation typically occurs when the patient's shoulder is forcefully abducted and externally rotated.

The diagnosis is made after review of the history and mechanism of injury and performance of a complete physical exam with imaging studies-the most critical component of diagnosis. ${ }^{4}$ Standard radiographs (anteroposterior, axillary, and scapular Y) can confirm the presence of a dislocation; once the diagnosis is confirmed, closed reduction of the joint should be performed. ${ }^{1}$ (Methods of reduction are beyond the scope of this article but have been recently reviewed. ${ }^{5}$ )

\section{Risk for recurrence}

\section{drives management choices}

Following an initial shoulder dislocation, the risk of recurrence is high. ${ }^{6,7}$ Rates vary based on age, pathology after dislocation, activity level, type of immobilization, and whether surgery was performed. Overall, age is the strongest predictor of recurrence: $72 \%$ of patients ages 12 to 22 years, $56 \%$ of those ages 23 to 29 years, and $27 \%$ of those older than 30 years experience recurrence. ${ }^{6}$ Patients who have recurrent dislocations are at risk for arthropathy, fear of instability, and worsening surgical outcomes. $^{6}$

Reducing the risk of a recurrent shoulder dislocation has been the focus of intense study. Proponents of surgical stabilization argue that surgery-rather than a trial of conservative treatment-is best when you consider the high risk of recurrence in young athletes (the population primarily studied), 
the soft-tissue and bony damage caused by recurrent instability, and the predictable improvement in quality of life following surgery.

In a recent systematic review and metaanalysis, there was evidence that, for firsttime traumatic shoulder dislocations, early surgery led to fewer repeat shoulder dislocations (number needed to treat $[\mathrm{NNT}]=2-4.7$ ). However, a significant number of patients primarily treated nonoperatively did not experience a repeat shoulder dislocation within 2 years. $^{2}$

The conflicting results from randomized trials comparing operative intervention to conservative management have led surgeons and physicians in other specialties to take different approaches to the management of shoulder dislocation..$^{2}$ In this review, we aim to summarize considerations for conservative vs surgical management and provide clinical guidance for primary care physicians.

\section{When to try conservative management}

Although the initial treatment after a traumatic anterior shoulder dislocation has been debated, a recent meta-analysis of randomized controlled trials showed that at least half of first-time dislocations are successfully treated with conservative management. ${ }^{2}$ Management can include immobilization for comfort and/or physical therapy. Age will play a role, as mentioned earlier; in general, patients older than 30 have a significant decrease in recurrence rate and are good candidates for conservative therapy. ${ }^{6}$ It should be noted that much of the research with regard to management of shoulder dislocations has been done in an athletic population.

\section{Immobilization may benefit some}

Recent evidence has determined that the duration of immobilization in internal rotation does not impact recurrent instability. ${ }^{8,9}$ In patients older than 30 , the rate of repeat dislocation is lower, and early mobilization after 1 week is advocated to avoid joint stiffness and minimize the risk of adhesive capsulitis. ${ }^{10}$

Arm position during immobilization remains controversial. ${ }^{11}$ In a classic study by Itoi et al, immobilization for 3 weeks in internal rotation vs $10^{\circ}$ of external rotation was associated with a recurrence rate of $42 \%$ vs $26 \%$, respectively. ${ }^{12}$ In this study, immobilization in $10^{\circ}$ of external rotation was especially beneficial for patients ages 30 years or younger. ${ }^{12}$

Cadaveric and magnetic resonance imaging (MRI) studies have shown external rotation may improve the odds of labral tear healing by positioning the damaged and intact parts of the glenoid labrum in closer proximity.$^{13}$ While this is theoretically plausible, a recent Cochrane review found insufficient evidence to determine whether immobilization in external rotation has any benefits beyond those offered by internal rotation. ${ }^{14} \mathrm{~A}$ recent systematic review and meta-analysis found that immobilization in external rotation vs internal rotation after a first-time traumatic shoulder dislocation did not change outcomes. ${ }^{2}$ With that said, most would prefer to immobilize in the internal rotation position for ease.

I More research is needed. A Cochrane review highlighted the need for continued research. ${ }^{14}$ Additionally, most of the available randomized controlled trials to date have consisted of young men, with the majority of dislocations related to sports activities. Women, nonathletes, and older patients have been understudied to date; extrapolating current research to those groups of patients may not be appropriate and should be a focus for future research. ${ }^{2}$

\section{Physical therapy: \\ The conservative standard of care}

Rehabilitation after glenohumeral joint dislocation is the current standard of care in conservative management to reduce the risk for repeat dislocation. ${ }^{15}$ Depending on the specific characteristics of the instability pattern, the approach may be adapted to the patient. A recent review focused on the following 4 key points: (1) restoration of rotator cuff strength, focusing on the eccentric capacity of the external rotators, (2) normalization of rotational range of motion with particular focus on internal range of motion, (3) optimization of the flexibility and muscle performance of the scapular muscles, and (4) increasing the functional sport-specific load on the shoulder girdle.
At least half of first-time dislocations are successfully treated with conservative management. 


\section{$>$}

An increasing number of dislocations portends a poor outcome with nonoperative treatment.
A common approach to the care of a patient after a glenohumeral joint dislocation is to place the patient's shoulder in a sling for comfort, with permitted pain-free isometric exercise along with passive and assisted elevation up to $100^{\circ} .{ }^{16}$ This is followed by a nonaggressive rehabilitation protocol for 2 months until full recovery, which includes progressive range of motion, strength, proprioception, and return to functional activities. ${ }^{16}$

More aggressive return-to-play protocols with accelerated timelines and functional progression have been studied, including in a multicenter observational study that followed 45 contact intercollegiate athletes prospectively after in-season anterior glenohumeral instability. Thirty-three of 45 (73\%) athletes returned to sport for either all or part of the season after a median 5 days lost from competition, with 12 athletes (27\%) successfully completing the season without recurrence. Athletes with a subluxation event were 5.3 times more likely to return to sport during the same season, compared with those with dislocations. ${ }^{17}$

Dynamic bracing may also allow for a safe and quicker return to sport in athletes ${ }^{18}$ but recently was shown to not impact recurrent dislocation risk. ${ }^{19}$

I Return to play should be based on subjective assessment as well as objective measurements of range of motion, strength, and dynamic function. ${ }^{15}$ Patients who continue to have significant weakness and pain at 2 to 3 weeks post injury despite physical therapy should be re-evaluated with an MRI for concomitant rotator cuff tears and need for surgical referral. ${ }^{20}$

\section{When to consider surgical intervention}

In a recent meta-analysis, recurrent dislocation and instability occurred at a rate of $52.9 \%$ following nonsurgical treatment. ${ }^{2}$ The decision to perform surgical intervention is typically made following failure of conservative management. Other considerations include age, gender, bone loss, and cartilage defect. ${ }^{21,22}$ Age younger than 30 years, participation in competition, contact sports, and male gender have been associated with an in- creased risk of recurrence. ${ }^{23-25}$ For this reason, obtaining an MRI at time of first dislocation can help facilitate surgical decisions if the patient is at high risk for surgical need. ${ }^{26}$

An increasing number of dislocations portends a poor outcome with nonoperative treatment. Kao et al demonstrated a second dislocation leads to another dislocation in $19.6 \%$ of cases, while $44.3 \%$ of those with a third dislocation event will sustain another dislocation. ${ }^{24}$ Surgery should be considered for patients with recurrent instability events to prevent persistent instability and decrease the amount of bone loss that can occur with repetitive dislocations.

\section{What are the surgical options?}

Several surgical options exist to remedy the unstable shoulder. Procedures can range from an arthroscopic repair to an open stabilization combined with structural bone graft to replace a bone defect caused by repetitive dislocations.

I Arthroscopic techniques have become the mainstay of treatment and account for $71 \%$ of stabilization procedures performed. ${ }^{21}$ These techniques cause less pain in the early postoperative period and provide for a faster return to work. ${ }^{27}$ Arthroscopy has the additional advantage of allowing for complete visualization of the glenohumeral joint to identify and address concomitant pathology, such as intra-articular loose bodies or rotator cuff tears.

I Open repair was the mainstay of treatment prior to development of arthroscopic techniques. Some surgeons still prefer this method-especially in high-risk groupsbecause of a lower risk of recurrent dislocation. ${ }^{28}$ Open techniques often involve detachment and repair of the upper subscapularis tendon and are more likely to produce long-term losses in external rotation range of motion. ${ }^{28}$

I Which one is appropriate for your patient? The decision to pursue an open or arthroscopic procedure and to augment with bone graft depends on the amount of glenoid and humeral head bone loss, patient activity level, risk of recurrent dislocation, and surgeon preference.

For the nonathletic population, the timing 


\section{$>$}

\section{Arthroscopic techniques have become the mainstay of treatment and account for $71 \%$ of stabilization procedures performed.}

of injury is less critical and surgery is typically recommended after conservative treatment has failed. In an athletic population, the timing of injury is a necessary consideration. An injury midseason may be "rehabbed" in hopes of returning to play. Individuals with injuries occurring at the end of a season, who are unable to regain desired function, and/or with periarticular fractures or associated full-thickness rotator cuff tears may benefit from sooner surgical intervention. ${ }^{21}$

Owens et al have described appropriate surgical indications and recommendations for an in-season athlete. ${ }^{21}$ In this particular algorithm, the authors suggest obtaining an MRI for decision making, but this is specific to in-season athletes wishing to return to play. In general, an MRI is not always indicated for patients who wish to receive conservative therapy but would be indicated for surgical considerations. The algorithm otherwise uses bone and soft-tissue injury, recurrent instability, and timing in the season to help determine management. ${ }^{21}$

\section{Outcomes:}

\section{Surgery has advantages ...}

Recurrence rates following surgical intervention are considerably lower than with conservative management, especially among young, active individuals. A recent systematic review by Donohue et al demonstrated recurrent instability rates following surgical intervention as low as $2.4 \% .^{29}$ One study comparing the outcome of arthroscopic repair vs conservative management showed that the risk of postoperative instability was reduced by $20 \%$ compared to other treatments. ${ }^{7}$ Furthermore, early surgical fixation can improve quality of life, produce better functional outcomes, decrease time away from activity, increase patient satisfaction, and slow the development of glenohumeral osteoarthritis produced from recurrent instability. ${ }^{2,7}$

Complications. Surgery does carry inherent risks of infection, anesthesia effects, surgical complications, and surgical failure. Recurrent instability is the most common complication following surgical shoulder stabilization. Rates of recurrent instability after surgical stabilization depend on patient age, activity level, and amount of bone loss: males younger than 18 years who participate in contact competitive sports and have significant bone loss are more likely to have recurrent dislocation after surgery. ${ }^{23}$ The type of surgical procedure selected may decrease this risk.

While the open procedures decrease risk of postoperative instability, these surgeries can pose a significant risk of complications. Major complications for specific open techniques have been reported in up to $30 \%$ of patients $^{30}$ and are associated with lower levels of surgeon experience. ${ }^{31}$ While the healing of bones and ligaments is always a concern, 1 of the most feared complications following stabilization surgery is iatrogenic nerve injury. Because of the axillary nerve's close proximity to the inferior glenoid, this nerve can be injured without meticulous care and can result in paralysis of the deltoid muscle. This injury poses a major impediment to normal shoulder function. Some procedures may cause nerve injuries in up to $10 \%$ of patients, although most injuries are transient. ${ }^{32}$

\section{Bottom line}

Due to the void of evidence-based guidelines for conservative vs surgical management of primary shoulder dislocation, it would be prudent to have a risk-benefit discussion with patients regarding treatment options.

- Patients older than $\mathbf{3 0}$ years and those with uncomplicated injuries are best suited for conservative management of primary shoulder dislocations. Immobilization is debated and may not change outcomes, but a progressive rehabilitative program after the initial acute injury is helpful. Risk factors for failing conservative management include recurrent dislocation, subsequent arthropathy, and additional concomitant bone or softtissue injuries.

I Patients younger than $\mathbf{3 0}$ years who have complicated injuries with bone or cartilage loss, rotator cuff tears, or recurrent instability, and highly physically active individuals are best suited for surgical management. Shoulder arthroscopy has become the mainstay of surgical treatment for shoulder dislocations. Outcomes are favorable and dislocation recurrence is low after surgical repair. Surgery does carry its own inherent 
risks of infection, anesthesia effects, complications during surgery, and surgical failure leading to recurrent instability.

JFP

\section{CORRESPONDENCE}

Cayce Onks, DO, MS, ATC, Penn State Hershey, Milton S. Hershey Medical Center, Penn State College of Medicine, Family and Community Medicine H154, 500 University Drive, PO Box 850, Hershey, PA 17033-0850; conks@ pennstatehealth.psu.edu

\section{References}

1. Lin K, James E, Spitzer E, et al. Pediatric and adolescent anterior shoulder instability: clinical management of first time dislocators. Curr Opin Pediatr. 2018;30:49-56.

2. Kavaja L, Lähdeoja T, Malmivaara A, et al. Treatment after traumatic shoulder dislocation: a systematic review with a network meta-analysis. Br J Sports Med. 2018;52:1498-1506.

3. Brelin A, Dickens JF. Posterior shoulder instability. Sports Med Arthrosc Rev. 2017;25:136-143.

4. Galvin JW, Ernat JJ, Waterman BR, et al. The epidemiology and natural history of anterior shoulder dislocation. Curr Rev Musculoskelet Med. 2017;10:411-424

5. Rozzi SL, Anderson JM, Doberstein ST, et al. National Athletic Trainers' Association position statement: immediate management of appendicular joint dislocations. J Athl Train. 2018;53:1117-1128.

6. Hovelius L, Saeboe M. Arthropathy after primary anterior shoulder dislocation: 223 shoulders prospectively followed up for twenty-five years. J Shoulder Elbow Surg. 2009;18:339-347.

7. Polyzois I, Dattani R, Gupta R, et al. Traumatic first time shoulder dislocation: surgery vs non-operative treatment. Arch Bone Jt Surg. 2016;4:104-108.

8. Cox CL, Kuhn JE. Operative versus nonoperative treatment of acute shoulder dislocation in the athlete. Curr Sports Med Rep. 2008;7:263-268.

9. Kuhn JE. Treating the initial anterior shoulder dislocation-an evidence-based medicine approach. Sports Med Arthrosc Rev. 2006;14:192-198.

10. Smith TO. Immobilization following traumatic anterior glenohumeral joint dislocation: a literature review. Injury. 2006;37: 228-237.

11. Liavaag S, Brox JI, Pripp AH, et al. Immobilization in external rotation after primary shoulder dislocation did not reduce the risk of recurrence: a randomized controlled trial. J Bone Joint Surg Am. 2011;93:897-904.

12. Itoi $E$, Hatakeyama $Y$, Sato $T$, et al. Immobilization in external rotation after shoulder dislocation reduces the risk of recurrence: a randomized controlled trial. J Bone Joint Surg Am. 2007;89: 2124-2131.

13. Miller BS, Sonnabend DH, Hatrick C, et al. Should acute anterior dislocations of the shoulder be immobilized in external rotation? A cadaveric study. J Shoulder Elbow Surg. 2004;13:589-592.
14. Hanchard NCA, Goodchild LM, Kottam L. Conservative management following closed reduction of traumatic anterior dislocation of the shoulder. Cochrane Database Syst Rev. 2014;(4) CD004962.

15. Cools AM, Borms D, Castelein B, et al. Evidence-based rehabilitation of athletes with glenohumeral instability. Knee Surg Sports Traumatol Arthrosc. 2016;24:382-389.

16. Lafuente JLA, Marco SM, Pequerul JMG. Controversies in the management of the first time shoulder dislocation. Open Orthop J. 2017;11:1001-1010.

17. Dickens JF, Owens BD, Cameron KL, et al. Return to play and recurrent instability after in-season anterior shoulder instability: a prospective multicenter study. Am J Sports Med. 2014;42 2842-2850.

18. Conti M, Garofalo R, Castagna A, et al. Dynamic brace is a good option to treat first anterior shoulder dislocation in season. Musculoskelet Surg. 2017;101(suppl 2):169-173.

19. Shanley E, Thigpen C, Brooks J, et al. Return to sport as an outcome measure for shoulder instability. Am J Sports Med. 2019;47:1062-1067.

20. Gombera MM, Sekiya JK. Rotator cuff tear and glenohumeral instability. Clin Orthop Relat Res. 2014;472:2448-2456.

21. Owens BD, Dickens JF, Kilcoyne KG, et al. Management of midseason traumatic anterior shoulder instability in athletes. $J \mathrm{Am}$ Acad Orthop Surg. 2012;20:518-526.

22. Ozturk BY, Maak TG, Fabricant P, et al. Return to sports after arthroscopic anterior stabilization in patients aged younger than 25 years. Arthroscopy. 2013;29:1922-1931.

23. Balg F, Boileau P. The instability severity index score. A simple preoperative score to select patients for arthroscopic or open shoulder stabilisation. J Bone Joint Surg Br. 2007;89:1470-1477.

24. Kao J-T, Chang C-L, Su W-R, et al. Incidence of recurrence after shoulder dislocation: a nationwide database study. J Shoulder Elbow Surg. 2018;27:1519-1525.

25. Porcillini G, Campi F, Pegreffi F, et al. Predisposing factors for recurrent shoulder dislocation after arthroscopic treatment. J Bone Joint Surg Am. 2009;91:2537-2542.

26. Magee T. 3T MRI of the shoulder: is MR arthrography necessary? AJR Am J Roentgenol. 2009;192:86-92.

27. Green MR, Christensen KP. Arthroscopic versus open Bankart procedures: a comparison of early morbidity and complications. Arthroscopy. 1993;9:371-374.

28. Khatri K, Arora H, Chaudhary S, et al. Meta-analysis of randomized controlled trials involving anterior shoulder instability. Open Orthop J. 2018;12:411-418.

29. Donohue MA, Owens BD, Dickens JF. Return to play following anterior shoulder dislocations and stabilization surgery. Clin Sports Med. 2016;35:545-561.

30. Griesser MJ, Harris JD, McCoy BW, et al. Complications and reoperations after Bristow-Latarjet shoulder stabilization: a systematic review. J Shoulder Elbow Surg. 2013;22:286-292.

31. Ekhtiari S, Horner NS, Bedi A, et al. The learning curve for the Latarjet procedure: a systematic review. Orthop J Sports Med. 2018;6:2325967118786930.

32. Shah AA, Butler RB, Romanowski J, et al. Short-term complications of the Latarjet procedure. J Bone Joint Surg Am. 2012;94: 495-501.

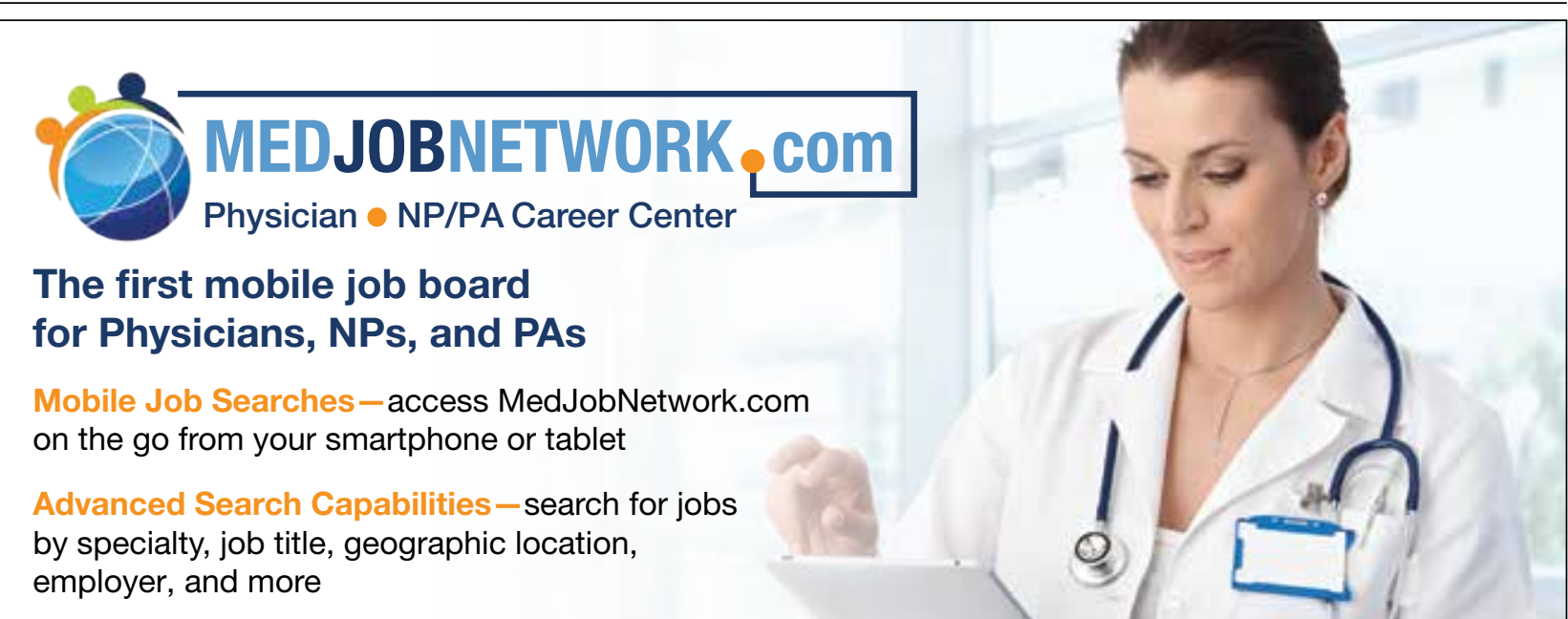

